\title{
ANÁLISE HISTÓRICA DOS PROJETOS DE INICIAÇÃO CIENTÍFICA SOBRE ÁGUA DESENVOLVIDOS EM UMA INSTITUIÇÃO DE ENSINO SUPERIOR NO ESTADO DO PARANÀ, BRASIL
}

\author{
HISTORICAL ANALYSIS SCIENTIFIC INITIATION PROJECTS ON WATER \\ DEVELOPED AT A HIGHER EDUCATION INSTITUTION IN PARANÁ, STATE, BRAZIL
}

\begin{abstract}
ANÁLISIS HISTÓRICA DE LOS PROYECTOS DE INICIACIÓN CIENTÍFICA SOBRE EL ÁGUA DESENVOLVIDOS EN UNA INSTITUCIÓN DE ENSEÑANZA SUPERIOR EN EL ESTADO DE PARANÁ, BRASIL
\end{abstract}

\author{
Maria de los Angeles Perez Lizama *,*** \\ maria.lizama@unicesumar.edu.br \\ Marcia Aparecida Andreazzi *,*** \\ marcia.andreazzi@unicesumar.edu.br
}

Edneia Aparecida de Souza Paccola*,*** edneia.paccola@unicesumar.edu.br

Luiz Felipe Machado Velho*,*** luiz.velho@unicesumar.edu.br

Arthur Gualberto Bacelar da Cruz Urpia**,*** arthut.urpia@unicesumar.edu.br

\footnotetext{
* Programa de Pós-Graduação em Tecnologias Limpas (PPGTL), Centro Universitário Cesumar/UNICESUMAR, Maringá-PR - Brasil ** Programa de Pós-Graduação em Gestão do Conhecimento nas Organizações (PPGCO), Centro Universitário Cesumar/UNICESUMAR, Maringá-PR - Brasil

*** Instituto Cesumar de Ciência, Tecnologia e Inovação/ICETI, Maringá-PR - Brasil
}

\begin{abstract}
Resumo
As pesquisas derivadas de projetos de iniciação científica buscam soluções para problemas em diferentes áreas do conhecimento e temáticas e, dentre essas temáticas, os recursos hídricos têm sido amplamente discutidos. Por isso, este trabalho analisou os projetos de iniciação científica desenvolvidos em uma Instituição de Ensino Superior privada, no sul do Brasil, durante um período de 15 anos, que abordaram diferentes aspectos relacionados à água. A avaliação histórica mostrou um número reduzido de projetos relacionados ao tema água $(3,23 \%)$, sendo que a maioria foi da categoria Programa de Iniciação Científica-PIC (60,3\%), desenvolvidos nos últimos 6 anos (74,8\%), principalmente pelos cursos de Ciências Biológicas (32,4\%), Engenharia Ambiental e Sanitária (29,8\%), Engenharia Civil (11,8\%;) e Engenharia Química (8,22\%), abordando temas como monitoramento, análise físico-química e/ou microbiológica em diferentes corpos hídricos e formas alternativas de tratamento de água e/ou efluentes. Baseados nos resultados desse estudo considera-se importante que as IES promovam ações de estímulo para a execução de mais estudos sobre o tema água, buscando propiciar aos alunos uma formação científica e acadêmica, que resulte em cidadãos sensibilizados com a importância da água para a vida, com a proteção dos recursos naturais e com o desenvolvimento sustentável.
\end{abstract}

Palavras Chave: Desenvolvimento sustentável. Meio ambiente. Pesquisa científica. Recursos hídricos.

\begin{abstract}
The scientific initiation searches solutions to problems in different areas of knowledge and themes, and among these themes, water resources have been widely discussed. Therefore, this study analyzed the scientific initiation projects developed in a private Higher Education Institution, south of Brazil, over a period of 15 years, which addressed different aspects related to water. The historical evaluation showed a small number of projects related to the theme water $(3.23 \%)$, most of them from the Scientific Initiation Program-SIP category (60.3\%), developed in the last 6 years (74.8\%), mainly by the Biological Sciences (32.4\%), Environmental and Sanitary Engineering (29.8\%), Civil Engineering (11.8\%;) and Chemical Engineering (8.22\%) courses and addressed topics such as monitoring, physicochemical analysis and / or microbiological treatment of water in different water resources and alternative forms of water and/or effluent treatment. Based on the results of this study, it is considered important that the HEIs promote actions to stimulate the execution of more studies on the theme of water, seeking to provide students with a scientific
\end{abstract}


and academic education that results in citizens sensitized with the importance of water for life with the protection of natural resources and sustainable development.

Keywords: Sustainabel development. Environment. Cientific research. Water resources.

\section{Resumen}

La iniciación científica investiga soluciones a problemas en diferentes áreas de conocimiento y temas, y entre estos temas, los recursos hídricos han sido ampliamente discutidos. Por lo tanto, este trabajo analizó los proyectos de iniciación científica desarrollados en una Institución de Enseñanza privada, en el sur de Brasil, durante un período de 15 años, que trataron diferentes aspectos relacionados con el agua. La evaluación histórica mostró un pequeño número de proyectos relacionados con el tema del agua (3.23\%), la mayoría de ellos de la categoría de programa de iniciación científica-PIC (60.3\%), desarrollados en los últimos 6 años (74.8\%), principalmente por los cursos de Ciencias biológicas (32.4\%), Ingeniería ambiental y sanitaria (29.8\%), Ingeniería civil (11.8\%) e Ingeniería química (8.22\%) y abordaron temas como monitoreo, análisis fisicoquímico y/o tratamiento microbiológico del agua en diferentes cuerpos hídricos y formas alternativas de tratamiento de aguas y/o efluentes. Con base en los resultados de este estudio, se considera importante que las IES promuevan acciones para estimular la ejecución de más estudios sobre el tema del agua, buscando brindar a los estudiantes una educación científica y académica que resulte en ciudadanos sensibilizados con la importancia del agua para la vida., con la protección de los recursos naturales y el desarrollo sostenible.

Palabras clave: Desarrollo sostenible. Medio ambiente. Investigación científica. Recursos hídricos.

\section{INTRODUÇÃO}

Atualmente existem várias modalidades de programas de iniciação científica (IC) ofertados aos alunos do ensino superior no Brasil, dentre eles o Programa de Bolsas de Iniciação Científica (PIBIC), o Programa Institucional de Bolsas de Iniciação em Desenvolvimento Tecnológico e Inovação Científica (PIBITI), o Programa Institucional de Bolsas de Iniciação Científica nas Ações Afirmativas (PIBIC-Af) e o Programa de Iniciação Científica e Mestrado (PICME) (CNPq, 2019), entretanto, as Instituições de Ensino Superior (IES) também podem ofertar outras modalidades. Mas, independentemente da modalidade, todos os programas almejam despertar jovens talentos para a ciência.

Os programas de IC sustentam a realização de pesquisas que contribuem para a formação profissional do aluno, envolvendo aspectos teóricos e metodológicos (BASTOS et al., 2010), melhoram a rotina de estudos, conferem uma estrutura curricular diferenciada, capacitam para a melhor expressão oral, escrita e para a leitura crítica (FAVA DE MORAES; FAVA, 2000), insere e fixa o discente no curso e na academia e contribui para o desenvolvimento do pensamento científico (CNPq, 2019). Todas estas vantagens também aumentam as possibilidades de o estudante ingressar na pós-graduação, tanto lato sensu quanto stricto sensu.

Ressalta-se, porém, que esses benefícios podem apresentar maiores dimensões de acordo com o tema escolhido para a pesquisa, sendo necessária uma escolha criteriosa baseada em problemas reais e atuais. Como exemplo, cita-se a escassez dos recursos naturais, principalmente dos recursos hídricos, os quais têm sido largamente discutidos nacional e internacionalmente. 
A busca pelo desenvolvimento sustentável no planeta, pautada no alcance dos Objetivos do Desenvolvimento Sustentável (ODS), inclui a preocupação com os recursos hídricos, a qual é apontada, de forma indireta, em vários objetivos, e de forma direta, nos ODS 6 e 14 (UNESCO, 2019). O objetivo 6 busca assegurar a disponibilidade e gestão sustentável da água e saneamento e elenca em suas metas, alcançar o acesso universal e equitativo a água potável e segura para todos; melhorar a qualidade da água; aumentar a eficiência do uso da água em todos os setores e assegurar retiradas sustentáveis e o abastecimento de água doce para enfrentar a escassez de água, e reduzir o número de pessoas que sofrem com a escassez de água; implementar a gestão integrada dos recursos hídricos em todos os níveis; proteger e restaurar ecossistemas relacionados com a água; ampliar a cooperação internacional e o apoio à capacitação para os países em desenvolvimento em atividades e programas relacionados à água e saneamento. Já o objetivo 14 relaciona a água à conservação e uso sustentável dos oceanos, dos mares e dos recursos marinhos para o desenvolvimento sustentável (UNESCO, 2019).

Dada a importância do cuidado com os recursos hídricos e toda a esfera de preocupações relacionadas a este tema, o Brasil publicou, em 8 de janeiro de 1997, a Lei № 9.433, que institui a Política Nacional de Recursos Hídricos (PNRH) e criou o Sistema Nacional de Gerenciamento de Recursos Hídricos (SINGREH) (BRASIL, 1997). Conhecida como Lei das Águas, a PNRH estabeleceu instrumentos para a gestão dos recursos hídricos de domínio federal e inovou com a instalação de comitês de bacias hidrográficas. A PNRH criou condições para identificar conflitos pelo uso das águas, por meio dos planos de recursos hídricos das bacias hidrográficas, e arbitrar conflitos no âmbito administrativo e deu maior abrangência ao Código de Águas, tornando a gestão dos recursos hídricos democrática (BRASIL, 1997).

Sendo assim, estudos sobre os diferentes aspectos relacionados ao tema água contribuem para o desenvolvimento sustentável. Brundtland (1987) afirmou que o desenvolvimento sustentável deve ser baseado no crescimento econômico, na proteção ao meio ambiente e na igualdade social, de modo a atender às necessidades do presente sem comprometer a capacidade de as gerações futuras satisfazerem as suas necessidades.

Essas investigações ocorrem principalmente nos meios acadêmicos, os quais apresentam como base as pesquisas de IC, portanto, pautados na relevante e atual questão relacionada aos recursos hídricos, o objetivo deste trabalho foi analisar uma série histórica de 15 anos, dos projetos de IC relacionados ao tema água, em uma IES privada brasileira. Analisar a evolução das pesquisas de IC relacionados a água permite identificar tendências e lacunas, e assim, nortear a elaboração de ferramentas e ações buscando contribuir com o aumento na quantidade e diversidade de pesquisas 
sobre o tema, resultando numa estratégia na busca pela sustentabilidade ambiental por meio das pesquisas científicas nas IES.

\section{PERCURSO METODOLÓGICO}

Esta pesquisa, caracterizada como estudo transversal quantitativo, analisou 4.052 projetos de IC cadastrados na base de dados da Diretoria de Pesquisa de uma Instituição de Ensino Superior, localizada em Maringá/ Paraná, entre os anos de 2004 a 2018 e selecionou os projetos de IC referentes ao tema "Água", totalizando 131 projetos aderentes ao tema.

Após a seleção, foram coletados os dados de: (a) ano de execução do projeto; (b) categoria do programa de IC e agência de fomento: PIBIC, financiado pelo Conselho Nacional de Desenvolvimento Científico e Tecnológico (CNPq) ou pela Fundação Araucária (FA); o PIBIT, financiado pelo CNPq ou pela Instituição; o PROBIC, financiado pela IES, e o PIC, que é um programa sem financiamento; (c) Grandes áreas do CNPq que o projeto está vinculado: agrárias, biológicas, engenharias, humanas, saúde e sociais aplicadas e (d) curso de graduação ao qual o projeto pertence.

Os dados foram tabulados, organizados em planilhas eletrônicas e avaliados por meio de estatística descritiva, que teve como foco revelar tendências e lacunas das pesquisas de iniciação científica envolvendo o tema água, na IES estudada.

\section{RESULTADOS E DISCUSSÕES}

Este estudo revelou uma restrita porcentagem de projetos desenvolvidos na IES no período avaliado, que pesquisaram sobre o tema "Água", somente 3,23\% (131/4.052) do total. Essa reduzida quantidade de projetos é crítica, em função da importância e da vasta abrangência da temática.

Evidenciando esta importância e abrangência, reportam-se os ODS, principalmente o ODS 6 que enfatiza sobre a necessidade de assegurar a disponibilidade e gestão sustentável da água e saneamento, envolvendo em suas metas o acesso a água potável, de qualidade e segura, o aumento da eficiência do uso da água, a redução do número de pessoas que sofrem com a escassez de água, a 
necessidade da gestão integrada dos recursos hídricos e da proteção e restauração dos ecossistemas relacionados com a água, dentre outros (UNESCO, 2019).

Ressalta-se que várias destas preocupações também aparecem na legislação brasileira, visto que a PNRH elenca, em seus objetivos, assegurar à atual e às futuras gerações a necessária disponibilidade de água com qualidade, a utilização racional e integrada dos recursos hídricos, a prevenção e a defesa contra eventos hidrológicos críticos de origem natural ou decorrentes do uso inadequado dos recursos naturais e incentiva e promove a captação, a preservação e o aproveitamento de águas pluviais (BRASIL, 2010). Dessa forma, dada às evidentes preocupações nacionais e internacionais, esperava-se um número maior de projetos sobre o tema.

A avaliação da evolução histórica (Figura 1), de acordo com o triênio de execução, permitiu identificar o número de projetos científicos, evidenciou que até 2012 o número projetos relacionados ao tema "Água" foi pequeno, contudo, a partir de 2012 esse número aumentou de forma expressiva (Figura 1), mostrando que 74,8 \% (98/131) dos projetos foram desenvolvidos nos últimos 6 anos.

Figura 1: Frequência (\%) de projetos de iniciação científica relacionados a "Água", desenvolvidos em uma IES privada entre os anos de 20042018.

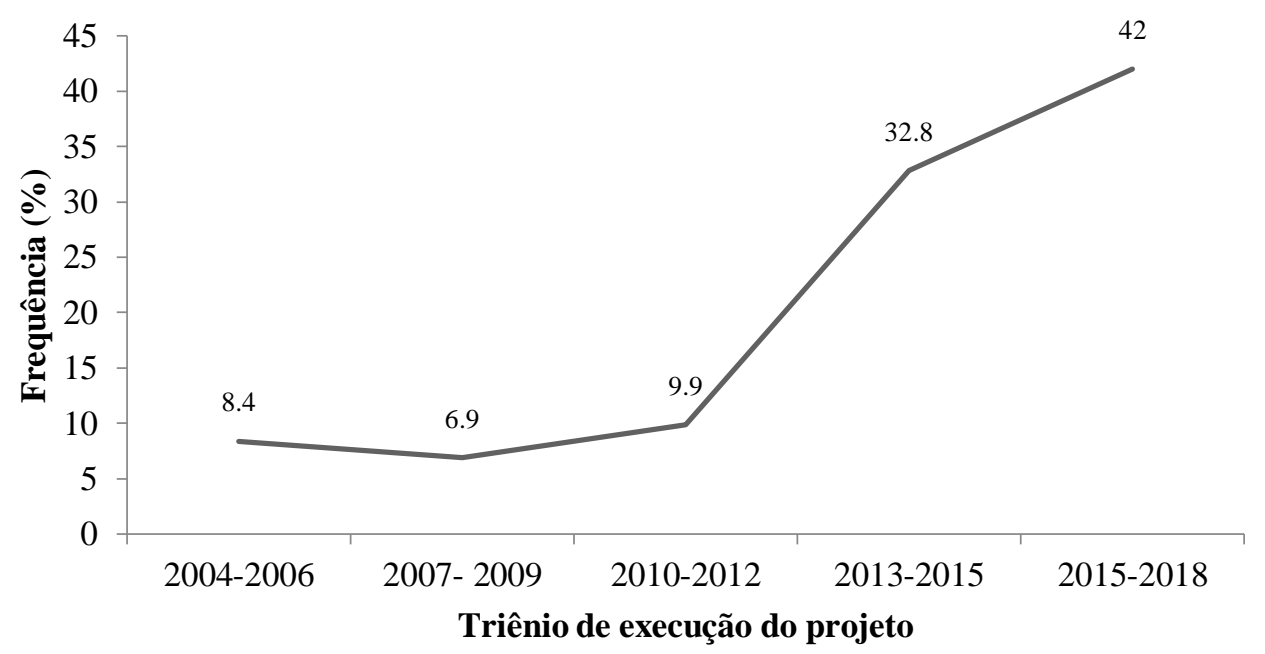

Atribui-se que, parte desse aumento na quantidade de projetos neste período esteja relacionado ao início das atividades na IES em estudo, do curso de graduação em Bacharelado em Ciências Biológicas (2011), o qual apresenta grande vínculo com o tema "Água”. Contudo, em 2014, a IES iniciou as atividades de um Programa de Pós-Graduação Stricto Sensu na área de Ciências Ambientais, que apresenta uma linha de pesquisa nominada Ecoefiência e Recursos Hídricos, o qual também pode ter contribuído com este aumento. Com relação à influência da pós-graduação sobre as temáticas ambientais, Pinheiro, Pinto e Cadete (2019) em um estudo que analisava a contribuição de um 
programa de mestrado para a educação ambiental e para o desenvolvimento local afirmaram que suas pesquisas revelaram-se conectadas às realidades e demandas e buscaram responder às dúvidas e questões que apoiaram a obtenção de elementos para a construção de uma proposta de intervenção educacional, socialmente inovadora e comprometida com o desenvolvimento local. Desta forma, verifica-se que de fato, uma pós-graduação exerce influência no meio no qual se insere e, no caso desse estudo, o mestrado influenciou, positivamente, o número de projetos sobre água na IES.

Assim, credita-se também ao maior volume de projetos sobre água o fato de que a IES em estudo é signatária dos Objetivos de Desenvolvimento Sustentável (ODS) e, portanto, estimula ações e projetos aderentes aos ODS e, no caso, o ODS 6, principalmente (UNESCO, 2019).

A avaliação da categoria de programa de iniciação científica (Figura 2) permitiu observar que a maior parte $(60,3 \%$; 79/131) dos projetos desenvolvidos foi do tipo PIC, ou seja, sem auxílio financeiro, seguido de projetos com auxílio institucional - PROBIC (24,4\%; 32/131).

Figura 2: Frequência (\%) de projetos de iniciação científica relacionados a "Água", desenvolvidos em uma IES privada entre os anos de 2004 e 2018, de acordo com a categoria.

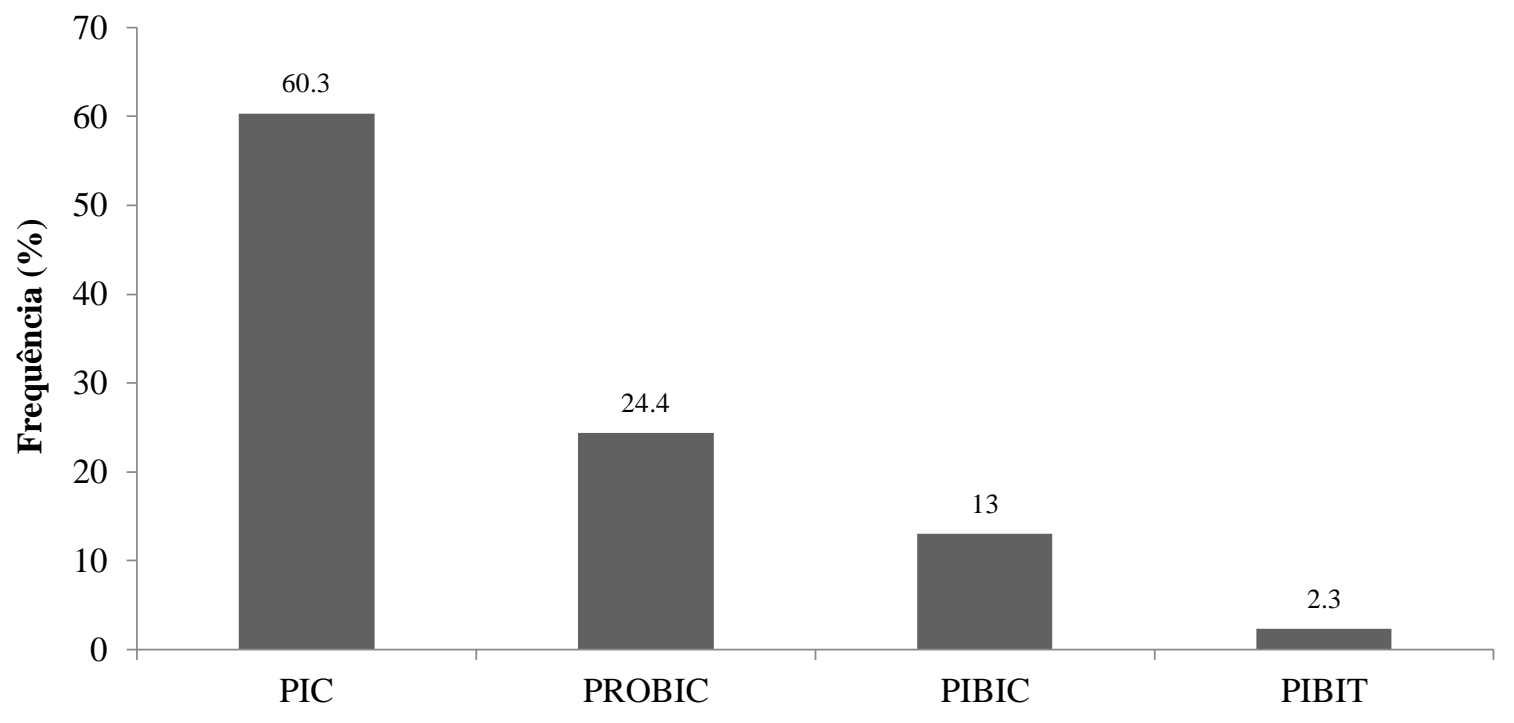

Modalidade de Programa de Iniciação Científica

$\mathrm{O} \mathrm{CNPq}$ categorizou as grandes áreas do conhecimento com o objetivo de proporcionar às instituições de ensino, pesquisa e inovação informações de forma rápida e sistemática sobre os projetos de pesquisa (CAPES, 2019). De fato, o emprego dessa classificação neste estudo, permitiu observar que $54,95 \%$ (72/131) dos projetos executados foram desenvolvidos por alunos da área de Engenharias, seguido de 32,06\% (42/131) da área de Biológicas (Figura 3). 
Buscando informações mais minuciosas, os dados foram analisados de acordo com os cursos de graduação envolvidos com o desenvolvimento dos projetos e, verificou-se que, o curso de graduação em Ciências Biológicas se destacou com a maior porcentagem de projetos $(32,4 \% ; 42 / 131)$, seguido do curso de Engenharia Ambiental e Sanitária (29,8\%; 39/131), Engenharia Civil (11,8\%; 15/131) e Engenharia Química $(8,22 \% ; 11 / 131)$ (Figura 4).

Figura 3: Frequência (\%) de projetos de iniciação científica relacionados a "Água", desenvolvidos em uma IES privada entre os anos de 2004 e 2018, de acordo com as grandes áreas do CNPq.

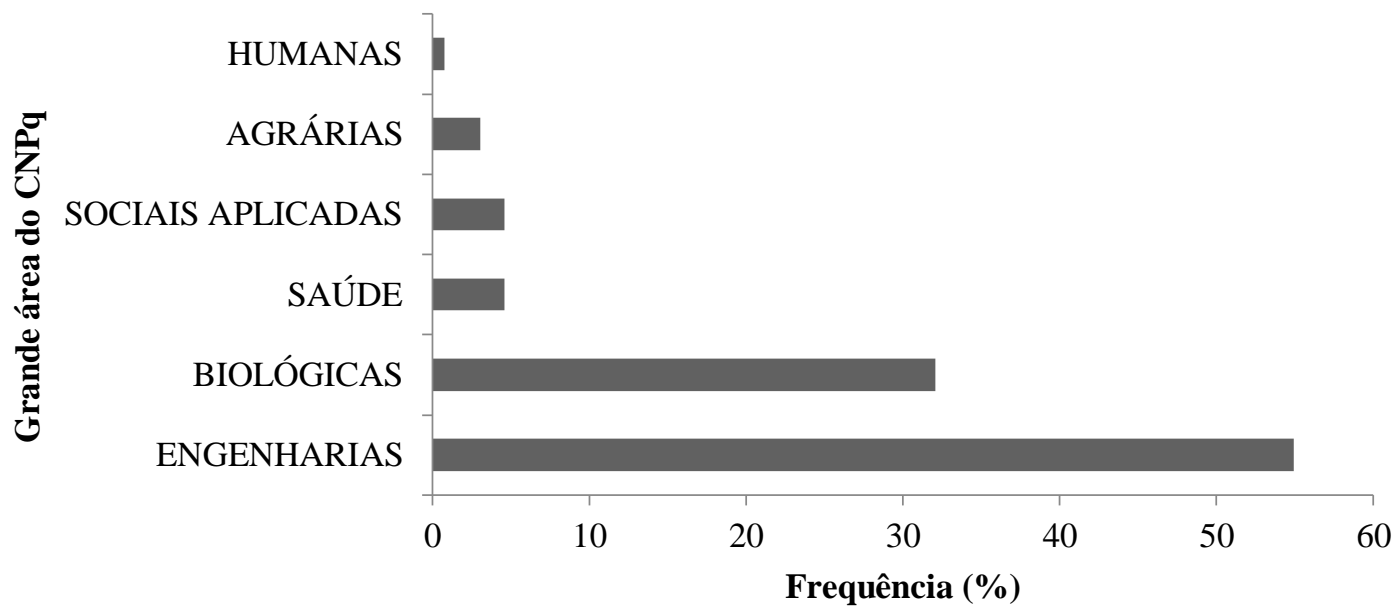

Figura 4: Frequência (\%) de projetos de iniciação científica relacionados a "Água", desenvolvidos em uma IES privada entre os anos de 2004 e 2018, de acordo com os cursos de graduação.

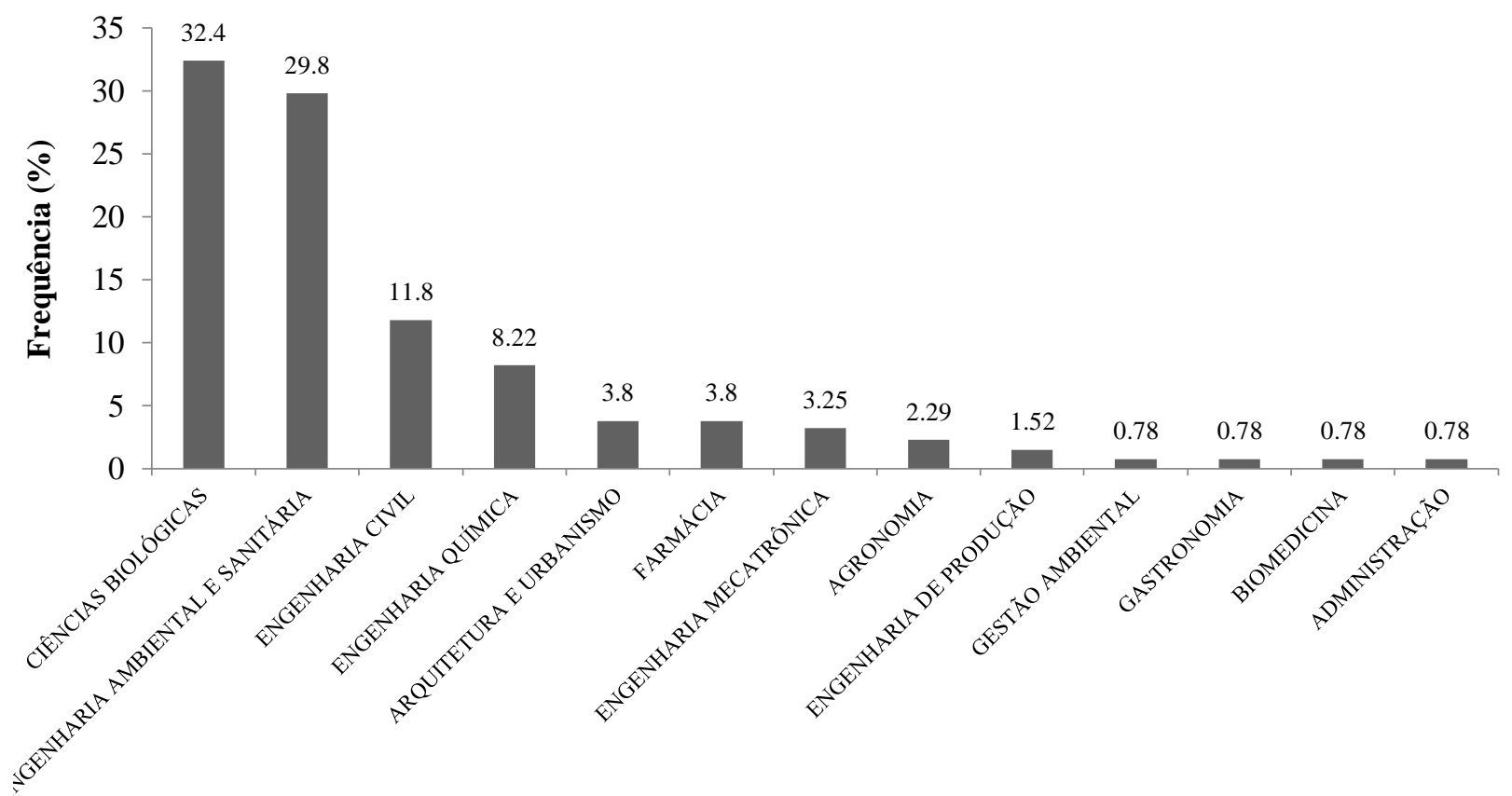

Curso de graduação de execução do projeto 
Os projetos executados pelo curso de Ciências Biológicas abordaram principalmente sobre temas como monitoramento, análise físico-química e/ou microbiológica da água em diferentes córregos, ribeirões e rios da região de Maringá. De forma complementar, os projetos desenvolvidos pelo curso de Engenharia Ambiental e Sanitária pesquisaram, além desses temas, formas alternativas de tratamento de água e/ou efluentes, empregando coagulantes ou Moringa oleifera (Figura 5). Para a organização e exposição desses resultados, foi utilizado um programa informático que produziu uma nuvem de palavras, organizando-as, em várias tamanhos, com base no número de menções feitas.

Figura 5: Nuvem de palavras dos eixos temáticos encontrados nos títulos dos projetos de iniciação científica aderentes ao tema “Água”, executados em uma IES privada, entre os anos de 2004 e 2018.

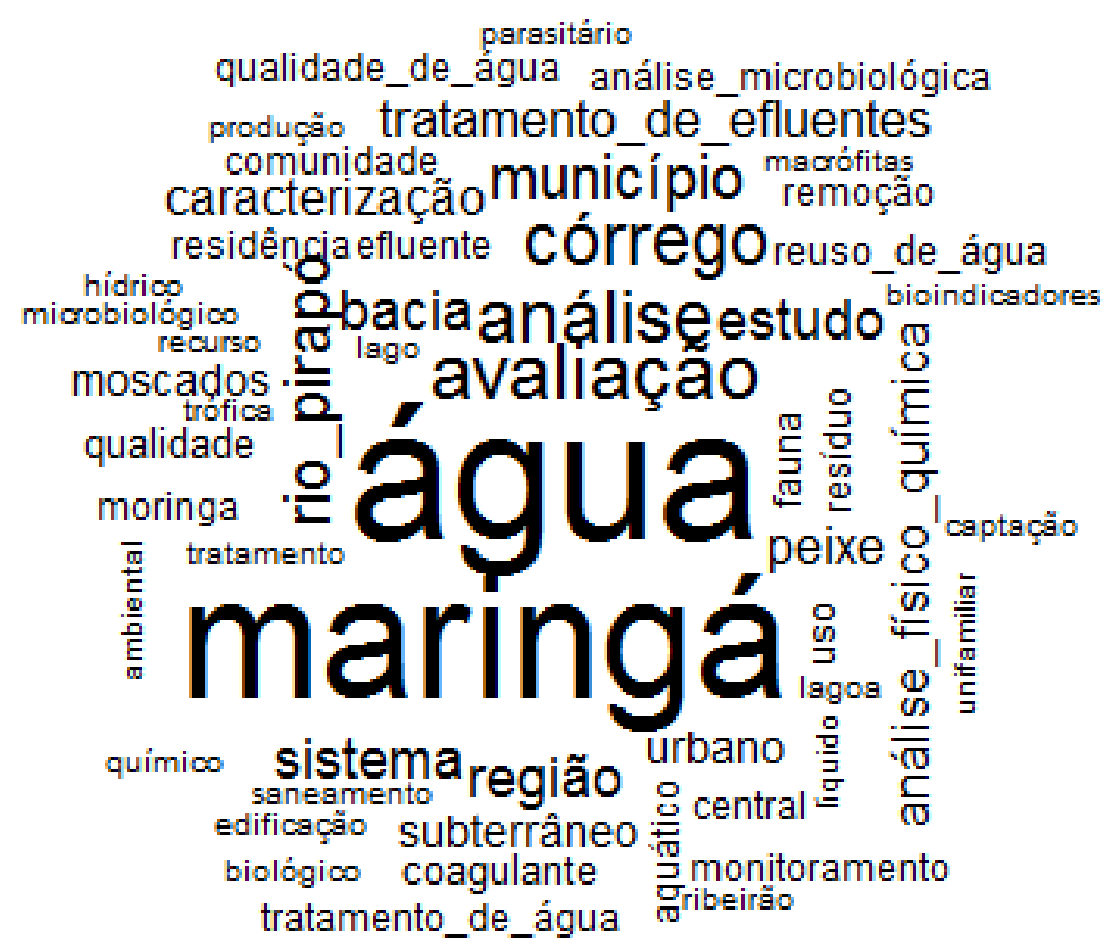

De fato, projetos que estudem sobre a análise e qualidade da água, em qualquer dimensão, são necessários e segue ao encontro da preocupação entre cientistas, Organizações não Governamentais (ONGs) e agências internacionais como a Organização das Nações Unidas (ONU), Organização das Nações Unidas para a Alimentação e a Agricultura (FAO), Organização das Nações Unidas para a Educação, Ciência e Cultura (UNESCO), em função dos elevados danos ao meio ambiente, aos organismos aquáticos e à saúde humana que a mesma pode provocar (CARVALHO et al., 2015). A água, fonte de vida e recurso limitado, vem sendo degradada em decorrência do aumento populacional e da poluição ambiental afetando milhares de pessoas no mundo, as quais apresentam algum tipo de 
enfermidade em função de sua baixa qualidade (MENDES et al., 2007). Desta forma, estudos sobre sua qualidade são imperativos.

Participar de programas de IC resulta em benefícios pessoais, acadêmicos e profissionais. Por certo, a participação dos estudantes de graduação em projetos de IC contribui para o desenvolvimento de várias competências e habilidades (MENEZES et al., 2013), cujas vantagens incluem estrutura curricular diferenciada e melhor capacitação para a expressão oral e escrita, leitura e habilidades manuais (FAVA DE MORAES; FAVA, 2000), redução no tempo de graduação, maior remuneração e maior possibilidade de ingressar na pós-graduação stricto sensu (CGEE, 2017), desenvolvimento do conhecimento e do pensamento científico (CNPq, 2019) além de possibilitar aprender, estruturar e desenvolver uma pesquisa científica (ANDRADE, 2019).

Contudo, considerando a atual atenção mundial aos recursos hídricos, infere-se que a fim de aumentar e diversificar a lista de vantagens que os programas de IC podem gerar, é fundamental aumentar a quantidade de projetos científicos que investiguem sobre temáticas e preocupações atuais, como é o caso da água. Desta forma, permitem contribuir com o necessário desenvolvimento sustentável, visto que, a falta de planejamento ambiental, o crescimento demográfico mundial, o crescente uso da água e do desmatamento em áreas de preservação e de mananciais, poderão resultar em sua escassez. Por isso, no mundo atual marcado pela degradação do meio ambiente, do ecossistema e dos recursos naturais, sobretudo da água, uma maior articulação desses temas nas instituições de ensino poderá contribuir com o ambiente e com a transformação social.

\section{CONSIDERAÇÕES FINAIS}

A análise histórica dos projetos de iniciação científica executados na IES avaliada, a partir de 2004, revelou uma pequena quantidade de projetos relacionados à água. Contudo, a partir de 2012, verificou-se um incremento nos estudos sobre esse tema. Observou-se também que a maioria dos projetos foi do tipo PIC, desenvolvidos por alunos dos cursos de Ciências Biológicas, Engenharia Ambiental e Sanitária e Civil e envolveram, principalmente, estudos sobre monitoramento, análise físico-química e/ou microbiológica e tratamento de água e/ou efluentes.

Baseado nos resultados desse estudo considera-se importante que as IES promovam ações de estímulo para a execução de mais estudos sobre o tema água, buscando propiciar aos alunos uma 
formação científica e acadêmica, que resulte em cidadãos sensibilizados com a importância da água para a vida, com a proteção dos recursos naturais e com o desenvolvimento sustentável. 


\section{Referências}

ANDRADE, R. O. Formação. Preparação para o futuro. Investir na iniciação científica durante a graduação pode ajudar estudantes a decidir sobre ingresso na pós. Pesquisa FAPESP. n. 277, p.96-97, 2019.

BASTOS, F.; MARTINS, F; ALVES, M.; TERRA, M.; LEMOS, C. S. A importância da Iniciação Científica para os alunos de graduação em Biomedicina. Revista Eletrônica Novo Enfoque, v. 11, n.11, p.61-66, 2010.

BRASIL. Lei $\mathbf{N}^{\mathbf{0}}$ 9.433, de 8 de janeiro de 1997. Política Nacional de Recursos Hídricos. Brasília, DF, 1997. Disponível em: <http://www2.camara.leg.br/legin/fed/lei/1997/l ei-9433-8-janeiro-1997-374778-normapl.html>. Acesso em: 29 jul. 2019.

BRUNDTLAND, G. H. Our common future: the world commission on environment and development. Oxford: Oxford University Press, 1987. 383p.

CARVALHO, K. Q.; LIMA, S. B.; PASSING, F. H.; GUSMÃO, L. K.; SOUZA, D. C.; KREUTZ, C.; BELINI, A. D.; ARANTES, E. J. Influence of urban area on the water quality of the Campo River basin, Paraná State, Brazil. Brazilian Jounal Biology, v.75, n.4, p. 96-106, 2015.

CENTRO DE GESTÃO E ESTUDOS ESTRATÉGICOS - CGEE. A Formação de novos quadros para CT\&I: avaliação do programa institucional de bolsas de iniciação. Brasília, DF. 2017. 175p. Disponível em: <https://www.cgee.org.br/documents/10195/734 063/2373_PIBIC_Relat\%C3\%B3rio_complcom .pdf>. Acesso em: 30 jul. 2019.

CONSELHO NACIONAL DE DESENVOLVIMENTO CIENTÍFICO E TECNOLÓGICO - CNPq. Disponível em: http://www.cnpq.br/web/guest/pibic/. Acesso em: 29 jul. 2019.

COORDENAÇÃO DE APERFEIÇOAMENTO DE PESSOAL DE NÍVEL SUPERIOR CAPES - disponível em: $<$ http://www.capes.gov.br/avaliacao/instrumento s-de-apoio/tabela-de-areas-do-conhecimentoavaliacao>. Acesso em: 24 jul. 2019.

FAVA DE MORAES, F.; FAVA, M. A iniciação científica: algumas vantagens e alguns riscos. São Paulo em Perspectiva. v.14, n.1, p.73-77, 2000.

MENDES, M. B., DE MIRANDA, M.G., DA SILVA, R. F., MARTINS, A. M. R., AVELAR, K. E. S. Carta da Terra e uma nova ética ambiental. In: MIRANDA M.G.; REZENDE, A. L.T; NERO, L. A.; MATTOS, M. R., BELOTI, V.; BARROS, M. A. F.; NETTO, D. P.; FRANCO, B. D. G. Organofosforados e carbamatos no leite produzido em quatro regiões leiteiras no Brasil: ocorrência e ação sobre Listeria monocytogenes e Salmonella spp. Ciência e Tecnologia de Alimentos, v. 27, n. 1, p. 201-204, 2007.

MENEZES, J. R.; CARPES, P. B. M.; GONÇALVES, R.; VIEIRA, A. S.; BARROS, W. M.; VARGAS, L. A Importância da Iniciação Científica para o aluno de Graduação. In: SALÃO INTERNACIONAL DE ENSINO, PESQUISA E EXTENSÃO, 5., 2013. Anais ... Bagé: Universidade Federal do Pampa, 2013 v.5, n.1, 2013.

PINHEIRO, M. B. C. F.; PINTO, J. M. N.; CADETE, M. M. M. Educação ambiental e desenvolvimento local: um estudo das contribuições do programa de mestrado. Revista Educação Ambiental em Ação, n.67, 2019.

ORGANIZAÇÃO DAS NAÇÕES UNIDAS PARA A EDUCAÇÃO A CIÊNCIA E A CULTURA - UNESCO - Agenda de Desenvolvimento Pós-2015 e os Objetivos de Desenvolvimento Sustentável. Disponível em: $<$ https://nacoesunidas.org/pos2015/>. Acesso em: 18 jul. 2019.

PAVANELLI, G. C.; EIRAS, A. C.; YAMAGUCHI, M. U.; TAKEMOTO, R. M. (org.). Zoonoses humanas transmissíveis por peixes no Brasil. Maringá: Cesumar, 2015. v. 1. 145p.

VASCONCELLOS, S. A. Zoonoses: conceito. CEVISA Online, 2013. Disponível em:

http://www.praiagrande.sp.gov.br/arquivos/cu rsos_sesap2/Zoonoses\%20Conceito.pdf. Acesso em: 08 maio 2018. 
ZANELLA, J. R. C. Zoonoses emergentes e reemergentes e sua importância para saúde e produção animal. Pesq. agropec. bras., Brasília, v. 51, n. 5, p. 510-519, maio 2016.

Recebido em: 20/09/2019

Aceito em: 09/11/2019

Endereço para correspondência:

Nome: Marcia Aparecida Andreazzi

Email: marcia.andreazzi@unicesumar.edu.br
DOI 10.1590/S0100-204X2016000500011.

Disponível em: http://www.scielo.br/scielo.php?script=sci_art text\&pid=S0100-

204X2016000500510\&lng=en\&nrm=iso. Acesso em: 08 maio 2018.

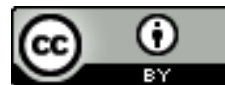

Esta obra está licenciada com uma Licença $\underline{\text { Creative }}$ Commons Atribuição 4.0 Internacional. 